\title{
Sustained immune tolerance induction in enzyme replacement therapy-treated CRIM-negative patients with infantile Pompe disease
}

Zoheb B. Kazi, ${ }^{1}$ Ankit K. Desai, ${ }^{1}$ Kathryn L. Berrier, ${ }^{1}$ R. Bradley Troxler, ${ }^{2}$ Raymond Y. Wang, ${ }^{3}$

Omar A. Abdul-Rahman, ${ }^{4}$ Pranoot Tanpaiboon, ${ }^{5}$ Nancy J. Mendelsohn, ${ }^{6}$ Eli Herskovitz,

David Kronn, ${ }^{8}$ Michal Inbar-Feigenberg, ${ }^{9}$ Catherine Ward-Melver, ${ }^{10}$ Michelle Polan, ${ }^{10}$ Punita Gupta, ${ }^{11}$ Amy S. Rosenberg, ${ }^{12}$ and Priya S. Kishnani'

'Division of Medical Genetics, Department of Pediatrics, Duke University Medical Center, Durham, North Carolina,

USA. ${ }^{2}$ University of Alabama at Birmingham, Birmingham, Alabama, USA. ${ }^{3}$ Division of Metabolic Disorders, Children's Hospital of Orange County, Orange, California, USA. ${ }^{4}$ Division of Medical Genetics, Department of Pediatrics, University of Mississippi Medical Center, Jackson, Mississippi, USA. ${ }^{5}$ Division of Cenetics and Metabolism, Children's National Health System, Washington, DC, USA. 'Genomics Medicine Program, Children's Hospitals and Clinics of Minnesota, Minneapolis, Minnesota, USA. ${ }^{7}$ Pediatric Endocrinology and Metabolism Unit, Soroka Medical Center, Beer Sheva, Israel. ${ }^{8}$ Department of Pediatrics, New York Medical College, Valhalla, New York, USA. 'Division of Clinical and Metabolic Genetics, The Hospital for Sick Children, University of Toronto, Toronto, Ontario, Canada. ${ }^{10}$ Division of Medical Genetics, Akron Children's Hospital, Akron, Ohio, USA. "Division of Medical Genetics, Department of Pediatrics, St. Joseph's Regional Medical Center Genetics, Paterson, New Jersey, USA. ${ }^{12}$ Division of Therapeutic Proteins, Office of Biotechnology Products, Center for Drug Evaluation and Research, US FDA, Bethesda, Maryland, USA.

BACKGROUND. Cross-reactive immunological material-negative (CRIM-negative) infantile Pompe disease (IPD) patients develop an immune response against enzyme replacement therapy (ERT) with alglucosidase alfa that nullifies ERT efficacy. Prophylactic immune tolerance induction (ITI) with rituximab, methotrexate, and IVIC successfully prevents development of deleterious rhGAA IgC antibodies; however, safety, likelihood of success, and long-term efficacy of ITI in a larger cohort remain unknown.

METHODS. Clinical data were analyzed for 19 CRIM-negative IPD patients who received ITI with rituximab, methotrexate, and IVIC in the ERT-naive setting (ERT+ITI) and compared to a historical cohort of 10 CRIM-negative IPD patients on ERT monotherapy.

RESULTS. ITI was safely tolerated, although infections were reported in 4 patients. Fourteen (74\%) ERT+ITI patients were alive, with a median age of $\mathbf{4 4 . 2}$ months at their final assessment. The eldest survivor was 103.9 months old, with 100.2 months of follow-up after initiation of ERT+ITI. Death (n = 5) occurred at a median age of $\mathbf{2 9 . 2}$ months and was unrelated to the administration of ITI. Fifteen

Authorship note: Z.B. Kazi and A.K. Desai are co-first authors.

Conflict of interest: P.S. Kishnani reports receiving research and grant support, honoraria, and consulting fees from Genzyme and is a member of the Pompe Disease and the Gaucher Disease Registry Advisory Boards.

Submitted: March 30, 2017

Accepted: June 6, 2017

Published: July 20, 2017

Reference information: JCI Insight. 2017;2(14):e94328 https://doi.org/10.1172/jici. insight.94328. patients either did not seroconvert $(n=8)$ or maintained low titers $(n=7$; defined as titers of $\leq 6,400$ throughout the course of ERT) following ERT+ITI. Only one patient developed high and sustained antibody titers (defined as titers of $\geq 51,200$ at or beyond 6 months on ERT). Left ventricular mass index (LVMI) decreased from a median of $248.5 \mathrm{~g} / \mathrm{m}^{2}$ at baseline to $76.8 \mathrm{~g} / \mathrm{m}^{2}$ at a median time from ERT+ITI initiation to 59 weeks. ERT+ITI significantly improved overall survival $(P=0.001)$, eliminated/reduced antibodies at values of $\leq 6,400$ at week 52 on ERT $(P=0.0004)$, and improved LVMI at week 52 on ERT $(P=0.02)$ when compared with ERT monotherapy.

CONCLUSION. Evidence from this international cohort of CRIM-negative IPD patients further supports the safety, feasibility, and efficacy of ITI in the prevention of immune responses to ERT.

TRIAL REGISTRATION. Clinicaltrials.gov NCT01665326.

FUNDING. This research was supported in part by the Lysosomal Disease Network, a part of NIH Rare Diseases Clinical Research Network, and by a grant from Genzyme, a Sanofi company. 


\section{Introduction}

The approval of insulin in 1982 as the first recombinant therapeutic protein marked the emergence of a new therapeutic era in which proteins, such as enzymes, hormones, cytokines, monoclonal antibodies, and toxins were approved to treat human disease. Despite this success, one of the challenges faced with administration of therapeutic proteins is the potential to elicit an immune response, which may directly affect safety and efficacy. Antibodies that develop against a therapeutic protein may alter its pharmacokinetic profile, diminish its pharmacodynamics, and compromise clinical benefits (1-6). Pompe disease (OMIM \# 232300, glycogen storage disease II) is an autosomal recessive lysosomal storage disorder treated via enzyme replacement therapy (ERT) with recombinant human acid $\alpha$-glucosidase (rhGAA, alglucosidase alfa), a treatment that has faced significant challenges posed by its immunogenicity in this patient population $(2,7)$. Variants in the $G A A$ gene lead to deficiency of acid $\alpha$-glucosidase (GAA, $\alpha$-1, 4-glucosidase), resulting in progressive accumulation of lysosomal glycogen, particularly in skeletal, cardiac, and smooth muscle tissues. Classical infantile Pompe disease (IPD), the most severe presentation, results in death secondary to cardiorespiratory failure within the first 2 years of life, if untreated (8)

ERT with rhGAA, currently the only FDA-approved treatment available for Pompe disease, has prolonged survival and improved clinical outcomes in patients with IPD (9-11). However, administration of rhGAA triggers an immune response in some patients, particularly those with 2 null GAA variants that cause complete absence of endogenous GAA on Western blot $(2,12)$ and are characterized as cross-reactive immunological material (CRIM) negative. The development of high and sustained rhGAA IgG antibody titers (HSAT; defined as titers of $\geq 51,200$ at or beyond 6 months on ERT) or sustained intermediate titers (SIT; defined as titers of $\geq 12,800$ and $<51,200$ within first year on ERT) is associated with subsequent clinical decline and death in patients with IPD, despite ongoing ERT (2, 13). Antibody-mediated reduction/diversion of active rhGAA to/from muscle tissues likely accounts for this therapeutic failure. Pharmacokinetic studies of ERT-treated patients with IPD demonstrated an average increase in clearance of infused rhGAA by $50 \%$ from week 1 to week 12 in patients with rhGAA IgG antibody titers of $\geq 12,800$ at week 12 (14).

Various approaches to prevent the development of rhGAA IgG antibody production in patients with Pompe disease in clinical and preclinical settings have been attempted, including a protocol with rituximab and sirolimus or mycophenolate, synthetic vaccine particles that contain only rapamycin, and anti-CD4 antibodies (15-20). The implementation of immune tolerance induction (ITI) using a combination of rituximab, methotrexate, and/or intravenous immunoglobulin (IVIG) infusions has been safely tolerated and effective in preventing the development of deleterious rhGAA IgG antibodies when administered in the ERT-naive and early ERT setting in patients with CRIM-negative IPD (2123). While implementation of ITI prior to initiation of ERT is ideal, patients who had received ITI after initiation of ERT and prior to the development of HSAT successfully tolerated ITI but required prolonged administration of rituximab, methotrexate, and IVIG infusions (22). Various combinations of rituximab, cyclophosphamide, IVIG, plasmapheresis, and increased doses of ERT have been tried without success in patients with IPD who developed HSAT prior to implementation of ITI. ITI (rituximab, methotrexate, and/or IVIG) with the addition of bortezomib, which targets antibody-secreting plasma cells, successfully reduced antibody titers and stabilized clinical decline in at least 4 patients, as previously published (24-26). However, such a "rescue" approach is not ideal, due to prolonged immunosuppression arising from the use of maintenance doses of rituximab and methotrexate along with bortezomib compared to a shorter 5-week course of ITI administered in an ERT-naive setting (26).

The administration of ITI in the ERT-naive setting has become the standard of care and optimal approach in treating patients with CRIM-negative IPD (27). We previously reported the successful implementation of ITI using rituximab, methotrexate, and IVIG in the first cohorts of CRIM-negative IPD patients on 2 and 9 patients who received ITI in the early ERT and ERT-naive settings, respectively (22, 23). The purpose of this study was to evaluate the long-term safety of prophylactic ITI in the ERT-naive setting (ERT+ITI) in a larger cohort. In addition, the effectiveness of ERT+ITI for CRIM-negative ERT-naive patients with IPD was compared to a historical cohort of CRIM-negative IPD patients on ERT monotherapy with regards to improvement in survival, prevention of rhGAA IgG antibody development, and reduction in left ventricular mass index (LVMI $\left.\left[\mathrm{g} / \mathrm{m}^{2}\right]\right)$. 
Table 1. Demographics of CRIM-negative IPD patients living at the time of database lock and treated with ERT+ITI in the ERT-naive setting

\begin{tabular}{|c|c|c|c|c|c|}
\hline Patient & Sex & Race/ethnicity & Age at diagnosis (mo) & Age at initiation of ERT (mo) & Current age (mo) \\
\hline 1 & $\mathrm{~F}$ & European descent & 1.9 & 3.7 & 103.9 \\
\hline 3 & $\mathrm{~F}$ & Hispanic & 2.5 & 3.0 & 67.7 \\
\hline 4 & M & African American & 3.5 & 4.5 & 66.9 \\
\hline 5 & $\mathrm{~F}$ & European descent & 2.0 & 2.5 & 64.2 \\
\hline 6 & $\mathrm{~F}$ & African American & 0.3 & 0.4 & 57.9 \\
\hline 10 & M & African American & 2.4 & 2.6 & 48.2 \\
\hline 14 & $\mathrm{~F}$ & European descent & 2.6 & 3.1 & 27.3 \\
\hline 15 & M & African American & 0.9 & 1.8 & 43.5 \\
\hline 16 & $\mathrm{~F}$ & French/English and Chinese & 5.9 & 0.9 & 22.1 \\
\hline 17 & $\mathrm{~F}$ & African American & 5.1 & 6.6 & 14.9 \\
\hline 18 & $\mathrm{~F}$ & Hispanic & Prenatal & 0.1 & 7.1 \\
\hline
\end{tabular}

IPD, infantile Pompe disease, ERT, enzyme replacement therapy; ITI, immune tolerance induction; F, female; M, male.

\section{Results}

Patient and ITI treatment details

Of the entire IPD cohort of 217 patients, 68 were identified as CRIM negative (31\%), based on Western blot and/or mutation status, as previously described (28). Of the 68 CRIM-negative patients, 19 fulfilled all inclusion criteria for the present study. The 10 CRIM-negative patients on ERT monotherapy used as a comparator group (described later) were a subset of these 68 patients. These CRIM-negative patients have been used as a comparator group in the earlier publications and, hence, for consistency the same group was used $(2,12,23)$. Patient demographics are presented in Tables 1 and 2, and the GAA variant data are presented in Supplemental Table 1 (supplemental material available online with this article; https://doi. org/10.1172/jci.insight.94328DS1). Thirty-eight GAA variants were identified, consisting of twenty-three $(60.5 \%)$ nonsense, twelve (31.5\%) frameshift, and $3(8.0 \%)$ splice-site variants. Nearly half of the identified $G A A$ variants $(n=17,45 \%$ ) were the common African American c. $2560 \mathrm{C}>\mathrm{T}$ variant. Two $G A A$ variants (c.1292_1295dupTGCA and c.437delT) were novel, likely pathogenic variants not previously described $(29,30)$. The median age at diagnosis was 2.6 months (range $0.0-9.8$ months), and median age at initiation of ERT+ITI was 3.4 months (range 0.1-10.9 months) (Tables 1 and 2). The median duration of data collection was 24.2 months since initiation of ERT+ITI (range 6.0-100.2 months).

Fourteen of the nineteen patients received one cycle of ITI and were off ITI medications for a median time of 32.9 months, with the longest duration being 99.2 months. The remaining 5 patients (patients $7,8,10,12$, and 15) received more than one cycle of ITI. Despite maintaining low antibody titers, patient 10 received maintenance rituximab every 2 to 3 months following ERT+ITI for 32 months. He has now been off all ITI medica-

Table 2. Demographics of deceased CRIM-negative IPD patients treated with ERT+ITI in the ERT-naive setting

\begin{tabular}{cccccc}
\hline Patient & Sex & Race/ethnicity & Age at diagnosis (mo) & Age at initiation of ERT (mo) & Age at death (mo) \\
2 & M & European descent & 2.4 & 3.6 & 5.9 \\
7 & F & African American & 3.0 & 3.9 & 25.4 \\
8 & F & Asian & 5.5 & 3.6 & 3.9 \\
9 & F & African American & 3.2 & 10.9 & 15.0 \\
12 & M & African American & 9.8 & 29.2
\end{tabular}

IPD, infantile Pompe disease, ERT, enzyme replacement therapy; ITI, immune tolerance induction; F, female; M, male. 
tions for 13.8 months. Per protocol, due to the development of antibody titers of 6,400 , patients 7 and 8 required a second cycle of ITI at 35 and 43 weeks from ERT+ITI initiation, respectively (23). Patients 7 and 8 were off all ITI medication for 11.6 and 12.4 months, respectively, at their final assessment. Patient 12 received a second cycle of ITI at 47 weeks following ERT+ITI initiation due to the development of antibody titers of $\geq 6,400$ and was on monthly maintenance rituximab and methotrexate at his final assessment. Patient 15 received a second and third cycle of ITI at 79 and 118 weeks following ERT initiation due to the development of antibody titers of 51,200 and 25,600, respectively. He was on monthly maintenance rituximab and methotrexate at his last assessment. Although planned, the treating physician was unable to get insurance approval for addition of bortezomib to the regimen at the time. No ITI regimen was withheld for safety concerns in any of the patients.

\section{Safety measures}

$B$ cell recovery. B cell recovery, as measured by normalization of CD19 percentage, was seen in all but one patient (patient 15) (Table 3) in which it was measured. Patient 15 was on rituximab at the final assessment prior to the time of data analysis; hence, B cell recovery was not expected. B cell counts were not assessed in patients 6 and 12, and the data on patient 13 was not available.

Vaccination and humoral immune response against vaccines. Seventeen patients $(90 \%)$ received routine immunizations and were up to date on their childhood immunizations at the time of data analysis. Patient 17 was not up to date on vaccines, and vaccination information was not available for patient 16 . Titers against routine vaccines were performed in 4 patients (patients 3, 11, 14, and 19) after B cell recovery to determine if rituximab administration affected the response to vaccines and were categorized as adequate (immune) or inadequate (not immune) based on reference values established by CLIA-certified laboratories. Patient 3 had an adequate humoral immune response to measles, mumps, and rubella (MMR); tetanus; Haemophilus influen$z a$ type $\mathrm{b}$; and pneumococcal serotypes $4,5,19 \mathrm{~F}, 23 \mathrm{~F}, 6 \mathrm{~B}, 11 \mathrm{~A}, 7 \mathrm{~F}, 9 \mathrm{~V}, 33 \mathrm{~F}, 1$, and 3 , while demonstrating an inadequate response to hepatitis $\mathrm{B}$ and pneumococcal serotypes $8,9 \mathrm{~N}, 12 \mathrm{~F}, 14,17 \mathrm{~F}, 20,22 \mathrm{~F}, 10 \mathrm{~A}, 15 \mathrm{~B}, 19 \mathrm{~A}$, and 2. Patient 11 had an adequate humoral immune response to MMR, tetanus, and diphtheria. Patient 14 had an adequate humoral immune response to MMR, varicella, diphtheria, polio, and some pneumococcal serotypes and demonstrated a lack of response to tetanus toxoid, Haemophilus influenza type b, hepatitis B, and certain pneumococcal polysaccharide antigens. Patient 19 started ERT+ITI at 3.3 months of age but had received vaccines against diphtheria, tetanus toxoid, pertussis, Haemophilus influenza type b, Pneumococcus, and hepatitis B prior to the start of the ITI regimen. B cell recovery was noted at age 8 months, and an adequate humoral immune response against these vaccines was maintained.

Infections around the time of ITI administration. Most patients safely tolerated ITI administration without infections. However, infections were reported in 4 patients (patients 3, 4, 14, and 16) (Table 3). Two patients (patients 3 and 14) experienced central line infections and bacteremia necessitating line removal and antibiotic treatment: patient 3 with Enterococcus faecalis, Pseudomonas fluorescens / putida, and Enterococcus raffinosus (ITI +2 months) and patient 14 with Klebsiella pneumoniae (ITI +3 months). The absolute neutrophil counts (ANCs) around the time of central line infections in patients 3 and 14 were $6,170 / \mu 1$ and 4,010/ $\mu 1$, respectively. Patient 4 experienced respiratory syncytial virus infection, and patient 16 suffered an episode of aspiration pneumonia and enterovirus/rhinovirus infection around the time of ITI administration. However, neither the ITI regimen nor ERT infusions were withheld for any subject due to infection.

$A N C, A S T$, and ALT data. ANC data were available for 10 patients at baseline and throughout the ITI administration. Six of these ten patients had an ANC of less than $750 / \mathrm{mm}^{3}$ following the ITI administration, which was reversible. Aspartate aminotransferase (AST) and alanine aminotransferase (ALT) data were available at baseline and throughout ITI administration in 11 patients. No increase in AST and ALT greater than 3 times their respective baseline values at the time of ITI administration was noted.

\section{Efficacy of ERT+ITI}

Survival. Of the 19 patients who were treated with ERT+ITI, 14 patients were alive at a median age of 44.2 months (range 7.1-103.9 months) and 5 patients (patients 2, 7, 8, 9, and 12) were deceased at a median age of 29.2 months (range 15.0-56.9 months) at the time of data analysis (Tables 1 and 2). The age at ERT+ITI initiation was significantly earlier in surviving patients $(P=0.03)$. Although the age at diagnosis was also earlier in surviving patients, this was not statistically significant $(P=0.13)$. In the 14 surviving patients, the median age at diagnosis and initiation of ERT+ITI was 2.5 months (range 0.0-5.9 months) and 2.8 months (range 0.1-6.6 months), respectively. In contrast, for the 5 deceased patients, 
Table 3. Safety measures and immune responses for ERT+ITI in CRIM-negative IPD patients treated in the ERT-naive setting

\begin{tabular}{|c|c|c|c|c|c|c|c|}
\hline Patient & Rounds of ITI & B cell recovery (CD19\%) & Infections & Vaccination & \multicolumn{3}{|c|}{ Antibody titers } \\
\hline & & & & & $\begin{array}{l}\text { Peak titers } \\
\text { (wk on ERT) }\end{array}$ & $\begin{array}{l}\text { Last titers } \\
\text { (wk on ERT) }\end{array}$ & $\begin{array}{c}\text { Immune tolerant } \\
\text { (Yes/No) }\end{array}$ \\
\hline \multicolumn{8}{|c|}{ CRIM-negative patients treated with ITI (alive at the time of database lock) } \\
\hline 1 & 1 & Yes & No & Yes & $1,600(38)$ & $200(103)$ & Yes \\
\hline 3 & 1 & Yes & $\begin{array}{c}\text { Enterococcus faecalis, } \\
\text { Pseudomonas } \\
\text { fluorescens/putida, } \\
\text { Enterococcus raffinosus }\end{array}$ & Yes & 0 & $0(281)$ & Yes \\
\hline 4 & 1 & Yes & RSV infection & Yes & 0 & $0(285)$ & Yes \\
\hline 5 & 1 & Yes & No & Yes & 0 & 0 (89) & Yes \\
\hline 6 & 1 & Not done & No & Yes & 0 & $0(70)$ & Yes \\
\hline 10 & $1^{\mathrm{A}}$ & Yes & No & Yes & 25,600 (199) & 25,600 (199) & No \\
\hline 11 & 1 & Yes & No & Yes & 0 & $0(214)$ & Yes \\
\hline 16 & 1 & Yes & $\begin{array}{l}\text { Aspiration pneumonia, } \\
\text { enterovirus/rhinovirus }\end{array}$ & NA & $200(81)$ & $200(81)$ & Yes \\
\hline 17 & 1 & Yes & No & No & $200(3)$ & 200 (32) & Yes \\
\hline 18 & 1 & Yes & No & Yes & 0 & $0(58)$ & Yes \\
\hline 19 & 1 & Yes & No & Yes & $3,200(20)$ & $3,200(24)$ & Yes \\
\hline \multicolumn{8}{|c|}{ Deceased CRIM-negative patients treated with ITI } \\
\hline 2 & 1 & Yes & No & Yes & 0 & $0(68)$ & Yes \\
\hline 7 & 2 & Yes & No & Yes & $6,400(31)$ & $6,400(59)$ & Yes \\
\hline 8 & 2 & Yes & No & Yes & $6,400(29)$ & $6,400(46)$ & Yes \\
\hline 9 & 1 & Yes & No & Yes & 1,600 (39) & $800(46)$ & Yes \\
\hline 12 & $2^{\mathrm{C}}$ & Not done & No & Yes & 25,600 (39) & $6,400(57)$ & Yes \\
\hline
\end{tabular}

IPD, infantile Pompe disease; ERT, enzyme replacement therapy; ITI, immune tolerance induction. ${ }^{\mathrm{A} P a t i e n t} 10$ received maintenance rituximab every 2 to 3 months after the completion of the first cycle. ${ }^{B}$ Patient 15 received monthly maintenance rituximab and methotrexate after the third cycle of ITI. ${ }^{\mathrm{C} P a t i e n t}$ 12 received maintenance rituximab and methotrexate until his final assessment.

the median age at diagnosis and initiation of ERT+ITI was 3.2 months (range 2.4-9.8 months) and 3.9 months (range 3.4-10.9 months), respectively (Tables 1 and 2). In all 5 deceased patients, the cause of death was respiratory failure due to disease progression. Of the 5 deceased patients, we were able to quantify CD19 percentage in 4 (patients 2, 7, 8, and 9), and all demonstrated B cell recovery (Table 3). Death occurred at a median time of 22.3 months (range 11.2-53.1 months) after completion of ITI and was unrelated to the administration of ITI medications.

rhGAA IgG antibody titers. Fifteen (79\%) of nineteen patients either tolerized (did not develop any rhGAA IgG antibodies) or maintained low titers (defined as titers of $\leq 6,400$ throughout the course of ERT) following ERT+ITI. Eight (42\%) of these fifteen patients never seroconverted after the implementation of ERT+ITI (Table 3) and continued to be antibody negative for a median of 79.5 weeks $(n=$ 8) following ERT+ITI initiation, with the longest available follow-up antibody titer data at 285 weeks. The remaining 7 (37\%) of these 15 patients seroconverted but maintained titers $\leq 6,400$ throughout the course of ERT. Four (21\%) patients (patients 10,12, 14, and 15) broke tolerance to the ERT and had antibody titers $>6,400$. Only 1 (patient 15 ) of these 4 patients developed HSAT, with titers peaking at 51,200 (range: 0-51,200). Three of these four patients (patients 10,12, and 14) developed SIT, with peak antibody titers of 25,600. Titers in patient 14 peaked at 25,600 (at ITI +22 months) but declined to 800 without additional ITI cycles after revision of rhGAA dosing from $40 \mathrm{mg} / \mathrm{kg}$ biweekly to 20 $\mathrm{mg} / \mathrm{kg}$ weekly. There was no significant difference in baseline characteristics between the patients who broke tolerance to the ERT (patients 10, 12, 14, and 15) as compared with the patients on ERT+ITI who maintained low/no titers. Overall, the median rhGAA IgG antibody titers at final assessment for the entire cohort was $200(n=19$; range $0-25,600)$ (Table 3$)$. 


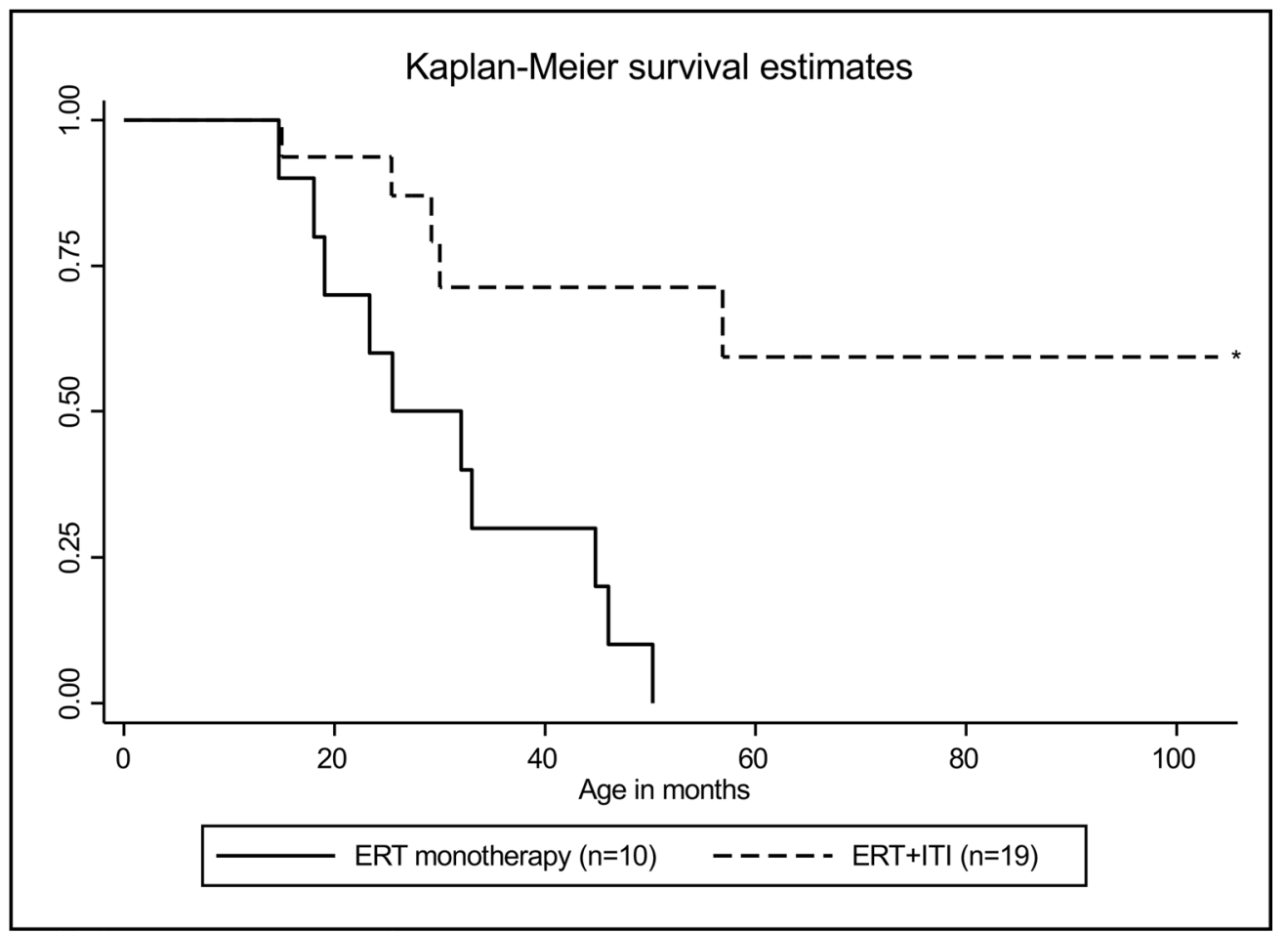

Figure 1. Kaplan-Meier curve for overall survival of CRIM-negative IPD patients treated with ERT+ITI versus those treated with ERT monotherapy. A significant difference in terms of overall survival $(P=0.001)$ was seen between the CRIM-negative ERT+ITI and the ERT monotherapy group. Death occurred in 5 patients in the ERT+ITI group at a median age of 29.2 months (range 15.0-56.9 months). There was a statistically significant difference ( $P=$ 0.03 ) when comparing age at ERT+ITI initiation, with the living patients starting ERT+ITI earlier, at a median age of 2.8 months, versus deceased patients, at a median age of 3.9 months. Survival data of patients with CRIM-negative IPD treated with ERT+ITI were analyzed using the Kaplan-Meier method, with 2-tailed $P$ values generated using the log-rank test (43).

Cardiac data. The upper limit of normal LVMI for infants is $64 \mathrm{~g} / \mathrm{m}^{2}$ ( $\geq 2 \mathrm{SD}$ higher than the age-appropriate normal mean) (31). Baseline LVMI was available on 16 patients, and follow-up LVMI was available on 18 patients. Median LVMI at baseline was $248.5 \mathrm{~g} / \mathrm{m}^{2}$ (range $65.4-508.0 \mathrm{~g} / \mathrm{m}^{2}$ ) (Table 4). The median LVMI at final assessment decreased to $76.8 \mathrm{~g} / \mathrm{m}^{2}\left(\right.$ range $48.0-424.2 \mathrm{~g} / \mathrm{m}^{2}$ ) at a median time of 59 weeks since ERT+ITI initiation.

\section{Comparison data of the CRIM-negative ERT+ITI group with ERT monotherapy group}

The baseline demographics and GAA variant data of the comparator group can be found in Supplemental Table 2. The age at diagnosis in the CRIM-negative ERT monotherapy group was significantly earlier than the ERT+ITI group $(P=0.01)$; however, ERT initiation time was comparable. The median age at ERT initiation in the CRIM-negative ERT monotherapy group was 3.3 months as compared with 3.4 months in the ERT+ITI group, with no significant statistical difference $(P=0.69)$. Of the 20 total $G A A$ variants in the ERT monotherapy group, $13(65 \%), 6(30 \%)$, and $1(5 \%)$ were nonsense, frameshift, and splice-site variants. $40 \%$ of the GAA variants were the common African American variant c. $2560 \mathrm{C}>\mathrm{T}$. The $G A A$ variant data and the age at ERT initiation in the CRIM-negative patients in the comparator group (ERT monotherapy) were similar to the ERT+ITI group.

\section{Survival}

All historical CRIM-negative patients who received ERT monotherapy (comparator group) were deceased at a median age of 28.8 months ( $n=10$; range 14.7-50.2 months) (13). Survival was significantly improved for patients in the CRIM-negative ERT+ITI group $(P=0.001)$ (Figure 1$)$. The age of the eldest survivor in the ERT monotherapy group was 50.2 months. Six patients in the CRIM-negative ERT+ITI group (patients 1, 2, 3, 4, 5, and 6) survived beyond the age of 50.2 months, with the eldest patient in the current cohort aged 103.9 months at time of data analysis. 


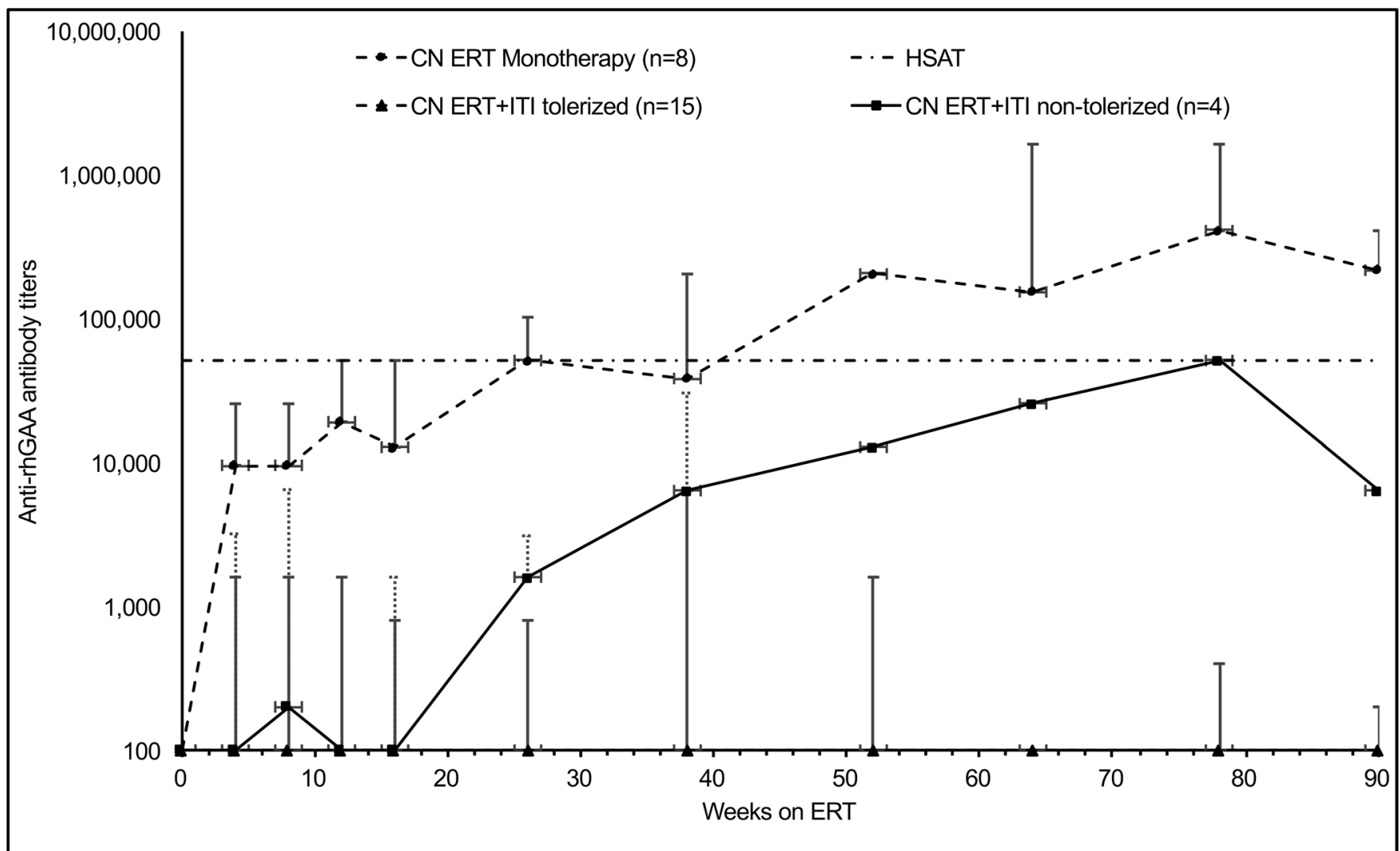

Figure 2. Comparison of longitudinal IgG antibody titers in CRIM-negative IPD patients treated with ERT+ITI tolerized, ERT+ITI nontolerized, or ERT monotherapy. High and sustained rhGAA IgG antibody titers (HSAT) defined as titers of $\geq 51,200$ at or beyond 6 months on ERT (shown by a horizontal dashed line). ERT+ITI tolerized $(n=15)$; nontolerized $(n=4)$; ERT monotherapy $(n=8)$. CN, CRIM-negative.

rhGAA IgG antibody titers

All CRIM-negative patients in the ERT monotherapy group $(n=8)$ and 11 of 19 patients in ERT+ITI group developed rhGAA IgG antibody titers. rhGAA IgG antibody titers were significantly lower in the ERT+ITI group at week $26(P<0.0001)$, week $52(P=0.0004)$, and week $78(P=0.0089)$ (Figure 2 and Supplemental Figure 1).

LVMI

The CRIM-negative ERT+ITI group ( $n=16$; median: $249 \mathrm{~g} / \mathrm{m}^{2}$; range: $\left.65-508 \mathrm{~g} / \mathrm{m}^{2}\right)$ had an insignificantly higher median LVMI at baseline as compared with the CRIM-negative ERT monotherapy group ( $n$ $=10$; median: $203 \mathrm{~g} / \mathrm{m}^{2}$; range: 89-308 g/m² $(P=0.39$; Figure 3$)$. For the first 26 weeks both groups had improvement in LVMI with an insignificant difference $(P=0.8)$, with a median of $111 \mathrm{~g} / \mathrm{m}^{2}(n=16$; range: $\left.55-444 \mathrm{~g} / \mathrm{m}^{2}\right)$ and $104 \mathrm{~g} / \mathrm{m}^{2}\left(n=10\right.$; range: $\left.57-269 \mathrm{~g} / \mathrm{m}^{2}\right)$ for ERT+ITI and ERT monotherapy groups, respectively. Subsequently, there was a continued reduction in LVMI in the ERT+ITI group, with a median of $88 \mathrm{~g} / \mathrm{m}^{2}\left(n=10\right.$; range $\left.59-144 \mathrm{~g} / \mathrm{m}^{2}\right)$ at week 52 , which was significantly different $(P=0.02)$ from the median of $129 \mathrm{~g} / \mathrm{m}^{2}\left(n=9\right.$; range $\left.63-201 \mathrm{~g} / \mathrm{m}^{2}\right)$ in the ERT monotherapy group, which worsened.

\section{Discussion}

Immune response to ERT is a significant predictor of therapeutic failure and ultimate death in ERT-treated patients with IPD. CRIM-negative cases from across the globe have demonstrated their susceptibility to the development of high rhGAA IgG titers $(2,7,32,33)$. However, unlike other prognostic factors, such as degree of existing damage at baseline, underlying genotype, differences in skeletal muscle fiber-type distribution, $A C E$ and $A C T N 3$ genotyping, and defective autophagy, immunogenicity is an avertable complication (34). We previously published our experience of ITI with methotrexate, rituximab, and IVIG in 


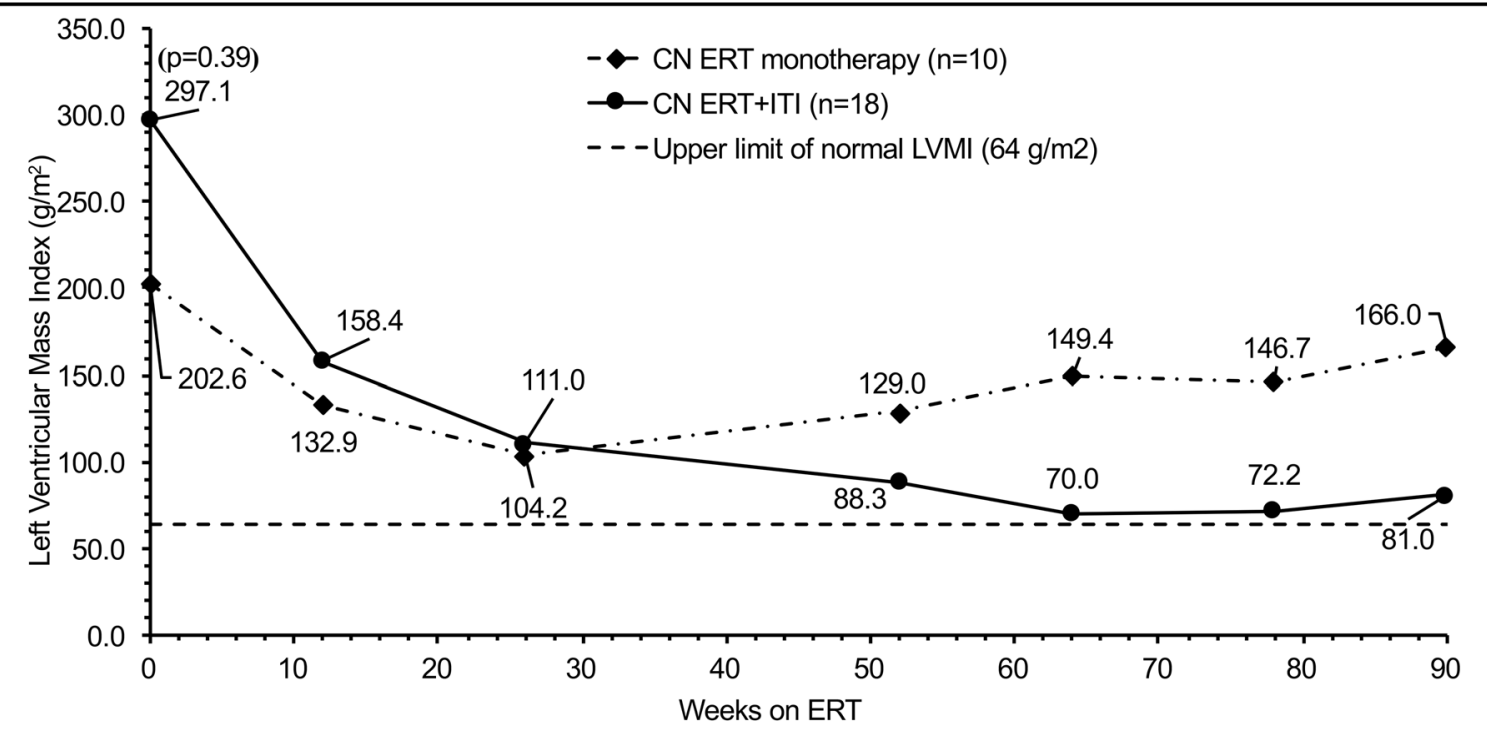

Figure 3. Comparison of longitudinal LVMI in CRIM-negative IPD patients treated with ERT+ITI versus those treated with ERT monotherapy. Left ventricular mass index (LVMI) continued to decline over time in the ERT+ITI cohort, while increasing after an initial period of reduction in the ERT monotherapy cohort. The upper limit of normal LVMI is $64 \mathrm{~g} / \mathrm{m}^{2}$ (represented by a horizontal dashed line). LVMI values were compared using Wilcoxon/Kruskal-Wallis rank-sum test. CN, CRIM-negative.

CRIM-negative IPD, in which the oldest patient was 58 months of age at the time of publication (22). This report of 19 patients is the largest cohort, to our knowledge, of ERT-treated CRIM-negative patients with IPD who completed at least one cycle of ITI. Data analysis included longitudinal outcomes up to 100.2 months since initiation of ERT+ITI. This represents a new cohort of CRIM-negative IPD patients, with an emerging clinical course distinct from the natural history of CRIM-negative IPD prior to the era of ITI (13). The purpose of this study was to characterize the long-term safety and efficacy of ITI in a unique cohort of long-term ERT-treated patients with CRIM-negative IPD.

Safety. Short-course ITI with prophylactic rituximab in the ERT-naive setting conveys a lower risk of adverse events than prolonged rituximab administration required in patients who develop HSAT. However, rituximab causes B cell suppression, resulting in an increased risk for infection. Therefore, IVIG is administered to provide some passive immunity. ITI was administered in this cohort without significant safety concerns, as evaluated with CD19 percentage as a marker of B cell recovery; vaccination status/titers against vaccines; infections around the time of ITI administration; and ANC, AST, and ALT data. CD19 percentage is associated with the degree of immune suppression and is a useful marker for monitoring the status of immune suppression but not immune tolerance. Of the patients in whom CD19 percentage was assessed, all but one patient who was still receiving ITI medications, demonstrated B cell recovery. These data suggest that ERT+ITI patients were able to achieve a B cell recovery after discontinuation of ITI medications, particularly rituximab, and were not in a constant state of immune suppression.

Seventeen patients received routine immunizations and were up to date on their childhood immunizations at the time of data analysis. Titers against the routine vaccines (MMR, tetanus, Haemophilus influenza type $\mathrm{b}$, pneumococcal conjugates, hepatitis $\mathrm{B}$, diphtheria, and varicella) were performed after $\mathrm{B}$ cell recovery in 4 patients. These patients maintained an adequate humoral immune response against the vaccine antigens, except for 2 patients, whose titers against vaccine showed an inadequate humoral immune response to hepatitis B, certain serotypes of Pneumococcus, tetanus toxoid, and Haemophilus influenza type $b$. Based on these data, it would be advisable to monitor vaccination titers after B cell recovery in patients who receive ITI (22) and consider additional vaccine boosts.

Although serious infections (i.e., Klebsiella, Pseudomonas, and Enterococcus) were seen in 4 of 19 patients, neither ITI nor ERT infusions were withheld for any patient. While these infections raise some concern about the use of ITI, bacterial infections were successfully treated with antibiotics and temporary removal of the patients' central lines. While this does confer some operative anesthesia risk due to cardiomyopathy, adherence to anesthesia precautions in Pompe patients resulted in positive 
Table 4. Efficacy of ERT+ITI in CRIM-negative IPD patients treated in the ERT-naive setting

\begin{tabular}{|c|c|c|c|c|c|c|c|c|}
\hline \multirow[t]{2}{*}{ Patient } & \multicolumn{2}{|c|}{ LVMI $\left(g / m^{2}\right)$} & \multicolumn{2}{|c|}{ Motor status } & \multicolumn{2}{|c|}{ Ventilation status } & \multicolumn{2}{|c|}{ Feeding status } \\
\hline & Baseline & $\mid \begin{array}{c}\text { Final } \\
\text { assessment } \\
\text { (wk on ERT) }\end{array}$ & Baseline & $\begin{array}{l}\text { Final assessment } \\
\text { (wk on ERT) }\end{array}$ & Baseline & $\begin{array}{l}\text { Final assessment } \\
\text { (wk on ERT) }\end{array}$ & Baseline & $\begin{array}{l}\text { Final } \\
\text { assessment } \\
\text { (wk on ERT) }\end{array}$ \\
\hline \multicolumn{9}{|c|}{ CRIM-negative patients treated with ERT+ITI (alive at time of database lock) } \\
\hline 1 & NA & NA & Hypotonia & Ambulatory (378) & No support & No support (378) & Oral & Oral (378) \\
\hline 3 & 160.3 & $70.5(303)$ & $\begin{array}{c}\text { Head lag, severe } \\
\text { hypotonia, motor delay }\end{array}$ & $\begin{array}{l}\text { Ambulatory, runs but } \\
\text { clumsy ( } 281)\end{array}$ & Oxygen & No support (281) & NG tube & Oral (281) \\
\hline 4 & 445.8 & $68.0(275)$ & $\begin{array}{c}\text { Head lag, antigravity } \\
\text { movements arms }> \\
\text { legs. }\end{array}$ & $\begin{array}{c}\text { Can move arms against } \\
\text { gravity (286) }\end{array}$ & Oxygen and BiPAP at night & $\begin{array}{l}\text { Invasively ventilated } \\
\qquad(271)\end{array}$ & NG tube & G tube $(271)$ \\
\hline 5 & 277.0 & $65.1(66)$ & $\begin{array}{c}\text { Severe hypotonia, } \\
\text { floppy baby, no head or } \\
\text { neck control }\end{array}$ & $\begin{array}{l}\text { Ambulates independently } \\
\text { (76) }\end{array}$ & Oxygen & No support (76) & NG tube & Oral (76) \\
\hline 6 & 409.6 & $92.3(53)$ & \begin{tabular}{|c|} 
Axial \\
hypotonia,withdraws \\
extremities to \\
stimulation, weak \\
grasp
\end{tabular} & $\begin{array}{c}\text { Sits with support, minimal } \\
\text { capacity for weight bearing } \\
\text { on lower extremities (53) }\end{array}$ & Invasively ventilated & $\begin{array}{l}\text { Invasively ventilated } \\
\text { (off ventilator } 10-12 \\
\text { hours a day) (58) }\end{array}$ & G tube & $G$ tube $(58)$ \\
\hline 10 & NA & $58.4(228)$ & Hypotonia & Ambulatory (199) & Invasively ventilated & No support (199) & $\mathrm{TP}$ & Oral (199) \\
\hline 11 & 140.6 & $61.7(194)$ & $\begin{array}{c}\text { Motor status and } \\
\text { milestones appropriate } \\
\text { for her age }\end{array}$ & Ambulatory (182) & No support & $\begin{array}{l}\text { CPAP with nasal mask } \\
\text { (199) }\end{array}$ & Oral & Oral (213) \\
\hline 13 & 508.0 & $245.0(11)$ & Hypotonia & NA & Invasively ventilated & Invasively ventilated (13) & TP & Oral (10) \\
\hline 14 & 156.7 & $52.6(96)$ & Hypotonia & $\begin{array}{c}\text { Ambulatory, shuffling/foot } \\
\text { slapping gait (105) }\end{array}$ & No support & No support (105) & Oral & Oral (105) \\
\hline 15 & 84.0 & $57.0(213)$ & Hypotonia & $\begin{array}{c}\text { Not ambulatory, can raise } \\
\text { hands to mouth, holds head } \\
\text { up, sits with and without } \\
\text { support (211) }\end{array}$ & No support & $\begin{array}{l}\text { Invasively ventilated } \\
\text { (204) }\end{array}$ & Oral & Oral (204) \\
\hline 16 & 176.0 & $48.0(103)$ & Hypotonia & NA & BiPAP & $\operatorname{BiPAP}(92)$ & G tube & G tube (92) \\
\hline 17 & 127.5 & $118.3(28)$ & Hypotonia & Not ambulatory (38) & Invasively ventilated & Invasively ventilated (38) & NG tube & G tube (38) \\
\hline 18 & 65.4 & $58.8(48)$ & $\begin{array}{l}\text { Head lag, central } \\
\text { hypotonia }\end{array}$ & $\begin{array}{l}\text { Normal developmental } \\
\text { milestones (58) }\end{array}$ & CPAP for a week & No support (37) & Oral & Oral (37) \\
\hline 19 & 433.1 & $113.4(22)$ & $\begin{array}{c}\text { Normal symmetric } \\
\text { bulk, appeared to have } \\
\text { normal tone }\end{array}$ & $\begin{array}{c}\text { Mild head lag, unable to sit } \\
\text { unsupported, attempting } \\
\text { to roll from back to front, } \\
\text { left to right, but is not } \\
\text { rolling completely without } \\
\text { assistance (10) }\end{array}$ & No support & No support (23) & Oral & G tube (23) \\
\hline \multicolumn{9}{|c|}{ Deceased CRIM-negative patients treated with ERT+ITI } \\
\hline 2 & NA & $257.0(64)$ & Hypotonia & \begin{tabular}{|} 
Prop-sit independently, \\
sits briefly without hand \\
support, rolls from supine \\
to side lying, bears weight \\
through lower extremities \\
in supported standing (80)
\end{tabular} & \begin{tabular}{|} 
Transient ventilation for \\
3 days
\end{tabular} & No support (67) & Oral & Oral (80) \\
\hline 7 & 317.2 & $144.0(54)$ & $\begin{array}{c}\text { Head lag unable to sit } \\
\text { or roll over }\end{array}$ & Standing with support (46) & Invasively ventilated & $\begin{array}{l}\text { Oxygen and BiPAP at } \\
\text { night (46) }\end{array}$ & NJ tube & $G$ tube (46) \\
\hline 8 & 347.1 & $108.0(36)$ & $\begin{array}{c}\text { Severe hypotonia, } \\
\text { antigravity movements } \\
\text { in arms }\end{array}$ & $\begin{array}{c}\text { Able to move arms against } \\
\text { gravity, near complete lower } \\
\text { extremity immobility (50) }\end{array}$ & Invasively ventilated & BiPAP at night (50) & NG tube & G tube (50) \\
\hline 9 & 220.0 & $83.0(39)$ & $\begin{array}{c}\text { Unable to } \\
\text { independently hold } \\
\text { hear or sit unsupported }\end{array}$ & $\begin{array}{c}\text { Not able to independently } \\
\text { hold head or sit } \\
\text { unsupported (46) }\end{array}$ & No support & Invasive ventilation (46) & NG tube & G) tube (46) \\
\hline 12 & 493.6 & $424.2(48)$ & $\begin{array}{c}\text { Unable to sit with } \\
\text { support, severe head } \\
\text { lag, rolled over at } 8 \\
\text { months }\end{array}$ & NA & Invasively ventilated & Invasively ventilated (57) & Oral & G tube (57) \\
\hline
\end{tabular}

IPD, infantile Pompe disease; ERT, enzyme replacement therapy; ITI, immune tolerance induction; LVMI, left ventricular mass index; NG tube, nasogastric tube; BiPAP, bilevel positive airway pressure; $\mathrm{G}$ tube, gastric tube; $\mathrm{G}$ tube, gastrojejunal tube; TP, transpyloric; CPAP, continuous positive airway pressure; NJ tube, nasojejunal tube. 
outcomes. ANC, AST, and ALT data were available for a subset of patients and demonstrated patient tolerance with a reversible decrease in ANC count ( 6 of 10 patients) and no elevation of greater than 3 times the baseline value for AST and ALT.

Five patients died during the course of follow-up, and none of these deaths were associated with the administration of ITI. Deceased patients had a later median age at diagnosis and initiation of ERT+ITI (3.2 months and 3.9 months, respectively) compared with earlier diagnosis and treatment for patients who were still alive at the completion of study (2.4 months and 2.6 months, respectively). ERT initiation was significantly earlier in surviving patients than patients now deceased $(P=0.02)$, highlighting the effect delayed treatment has on baseline disease severity and the need for timely diagnosis and treatment).

Efficacy. ERT+ITI effectively prevented immunogenicity, reduced cardiomyopathy, and improved survival for CRIM-negative patients with IPD. LVMI is an easily assessed measure of cardiomyopathy that has been used in pivotal clinical trials to assess efficacy of alglucosidase alfa (9-11). CRIM-negative patients with IPD treated with ERT monotherapy demonstrated initial improvement of LVMI within the first 6 months of treatment, followed by subsequent worsening due to the development of $\operatorname{HSAT}(24,25)$. In contrast, CRIM-negative IPD patients treated with ERT+ITI showed continued improvement of LVMI, nearly reaching the upper limit of normal $\left(64 \mathrm{~g} / \mathrm{m}^{2}\right)(31)$.

As previously reported in our historical cohort of CRIM-negative IPD patients on ERT monotherapy, all were either deceased or invasive ventilation dependent by 27.1 months of age, and the median age of death was 28.8 months ( $n=10$; range 14.7-50.2 months) (13). In contrast, in the present cohort of ERT+ITI-treated patients, 12 of 19 patients survived beyond 28.8 months (median age of 44.2 months), with a statistically significant $(P=001)$ difference in overall survival in comparison to the historical cohort of CRIM-negative IPD patients on ERT monotherapy. The details in Table 4 show that 7 CRIM-negative patients were ambulatory, 8 patients needed no ventilator support, and 10 patients were feeding orally at their final assessment. This clearly demonstrates that ERT +ITI has allowed these patients to be ambulatory, free of invasive ventilation, and to behave like CRIM-positive survivors on ERT.

Fifteen patients had low or no rhGAA IgG antibody titers after implementation of ERT+ITI. Of the 4 patients who developed titers during the study period, only one patient developed HSAT. Although there are 3 cases previously reported describing CRIM-negative patients who did not develop HSAT without ITI, one patient received omalizumab, an IgG monoclonal antibody that binds to IgE, for a severe allergic reaction to alglucosidase alfa; the other sibling pair had a splice site mutation in heterozygosity $(35,36)$. A role for omalizumab in preventing the development of an IgG response cannot be excluded in this case. Therefore, we believe that CRIM-negative ERT monotherapy cases who did not develop HSAT represent a smaller subset compared with CRIM-negative cases reported from across the globe with higher susceptibility to the development of high rhGAA IgG titers $(2,7,32,33)$. Currently, there is no way to predict CRIM-negative cases who would not develop HSAT/SIT, and this can be a confounding factor while interpreting the efficacy of this ITI protocol. Prior pharmacokinetic studies showed that patients with antibody titers of $\geq 12,800$ at week 12 had an average increase in clearance of 50\% from week 1 to week 12 (14). Ultimately, this supports the assumption that development of antibody titers negatively affects clinical outcomes. Prevention of an antagonistic immune response, as illustrated in the cohort presented here, results in greater efficacy of ERT, as demonstrated by improved LVMI and overall survival. Most patients $(n=14)$ only required one round of ITI. The need for additional rounds of ITI with the same regimen may partly be due to a Fc- $\gamma$ receptor polymorphism, which reduces the efficacy of rituximab (37). This requires further investigation and may warrant consideration for use of other B cell-suppressive agents. Because rituximab is a monoclonal antibody, administering IVIG prior to rituximab may saturate the FcRn receptor, thereby precluding recycling of rituximab and its sustained activity (38). Thus, timing of drug administration is critical and requires further exploration. Nevertheless, based on current data and clinical practices, we recommend rituximab administration prior to IVIG.

Although results from the present cohort provide evidence in support of ERT+ITI for patients with CRIM-negative IPD, additional research and protocol adjustments are necessary moving forward. The ITI regimen utilized here targets B and T cells in a nonspecific manner, highlighting the need for development of antigen-specific tolerance induction. Additional challenges with the use of this ITI protocol include the high cost and lack of availability of rituximab in certain countries as well as safety issues concerning infection risks due to B cell suppression, as mentioned above. The next step toward understanding the immune mechanistic data is to study the detailed immune phenotyping of patients that receive the ITI protocol this is being planned. 
Conclusions. Ultimately, longitudinal data from the present cohort demonstrate that treatment with ERT+ITI is safe and efficacious, and it allows CRIM-negative IPD patients to continue to maintain clinical benefit by reducing or eliminating substantial antibody titers over time with ongoing ERT, as compared with CRIM-negative IPD patients treated with ERT monotherapy. ERT+ITI results in tolerance, as evidenced by low antibodies to the rhGAA after B cell recovery while maintaining titers against the administered vaccines in a majority of patients. As such, the implementation of ITI is associated with improved overall survival and normalization of LVMI, which further support our previous experience with CRIM-negative IPD patients treated with ERT+ITI $(22,23)$. With the inclusion of Pompe disease in the US Recommended Uniform Screening Panel and international newborn screening panels, IPD is increasingly identified within the newborn period, allowing timely treatment. This is particularly important, as highlighted by our finding that patients diagnosed and initiated on ERT+ITI treatment later in time have poorer outcomes due to advanced disease. ITI utilizing rituximab, methotrexate, and IVIG should be implemented as standard of care in the management of CRIM-negative patients with IPD.

\section{Methods}

Patients. Retrospective data were collected from an international cohort of patients with IPD, totaling 217 at the time of this report, who were enrolled in a Duke IRB-approved protocol (protocol 00001562 , LDN6709 site 206; ref. 39).

From this cohort, consecutive participants were identified for inclusion in the present study based on the following criteria: (a) a confirmed diagnosis of CRIM-negative IPD (10); (b) initiation of ITI in the ERT-naive state, with successful completion of at least one cycle of ITI with rituximab (4 weekly doses intravenous1y), methotrexate (3 doses per week within the first 3 ERT infusions subcutaneously/orally), and/or IVIG (monthly) (Supplemental Figure 2) (23); (c) ERT with alglucosidase alfa at a cumulative dose of 20-40 mg/kg weekly or biweekly; and (d) at least 6 months of follow-up data since initiation of ERT+ITI.

A cohort of CRIM-negative IPD patients who participated in the original clinical trials of rhGAA, prior to the establishment of ITI as standard of care, served as a comparison group $(10,11,40,41)$. A total of 10 CRIM-negative IPD patients treated with ERT monotherapy were identified, and their overall survival data were reviewed along with their rhGAA IgG antibody titers and LVMI at baseline, week 26, and week 52 on ERT, for comparison with CRIM-negative IPD patients on ERT+ITI.

Data collection. Relevant clinical data, including GAA variants, CRIM status, and rhGAA IgG antibody titers (determined by Genzyme) were extracted from medical records of qualifying patients (10, 11, 28, 42). Longitudinal data, provided by the onsite local treating physician, were collected with regard to rhGAA IgG antibodies, LVMI, motor status, pulmonary status (ventilator use), feeding status (oral feeds, gastrostomy tube, nasogastric tube) at baseline and follow-up as possible. For deceased patients, age and cause of death were collected. LVMI was measured by 2D, M-mode, and Doppler echocardiography. ITI safety was assessed by measuring CD19 percentage as a marker of B cell recovery. Flow cytometry was used to assess CD19 percentage using standard methods. Vaccination status and antibody response to vaccines were collected as markers of B cell recovery and to ascertain if administration of rituximab resulted in loss of protective immunity. ITI safety was also assessed by collecting data on infections around the time of ITI administration and ANC, AST, and ALT levels before and after completion of ITI. AST and ALT values 3 times above baseline and ANC values of less than 750/ $\mathrm{mm}^{3}$ were considered an adverse event related to ITI. Data collection continued until April 2016 or until at least 6 months had passed since initiation of ERT+ITI, at which time the database was locked for analysis.

Statistics. Survival data for patients with CRIM-negative IPD treated with ERT+ITI were analyzed using the Kaplan-Meier method, with 2-tailed $P$ values generated using the log-rank test (43), and compared to a historical cohort of CRIM-negative patients with IPD on ERT monotherapy (23). IgG antibody titers and LVMI values were compared using Wilcoxon/Kruskal-Wallis rank-sum test $(2,12)$. Other nonparametric variables were analyzed using Kruskal-Wallis Rank sum test. Analyses were performed with STATA version 14.2 (StataCorp LP). Descriptive data are presented as medians due to the limited sample size.

Study approval. This study was conducted according to the principles of the Declaration of Helsinki and approved by the Duke University Medical Center IRB (protocol 00001562). Patients were included in the study after provision of written informed consent by their legally authorized representative. One patient (patient 1) was enrolled after approval from the IRB or ethics committee approval at Soroka University Medical Center. All the other patients were enrolled in the Duke University Medical Center IRB (protocol 00001562). 


\section{Author contributions}

Conception and study design were performed by ZBK, KLB, and PSK. Development of methodology was performed by ZBK, KLB, and PSK. Acquisition of data was performed by ZBK, AKD, KLB, RBT, RYW, OMAAR, PT, NJM, EH, DK, MIF, CWM, MP, PG, and PSK. Analysis and interpretation of data were performed by ZBK, AKD, KLB, and PSK. Writing, review, and/or revision of the manuscript were done by ZBK, AKD, KLB, RBT, RYW, OMAAR, PT, NJM, EH, DK, MIF, CWM, MP, PG, ASR, and PSK.

\section{Acknowledgments}

This research was supported by a grant from Genzyme, a Sanofi company (Cambridge, Massachusetts, USA), and supported in part by the Lysosomal Disease Network, a part of NIH Rare Diseases Clinical Research Network. The Lysosomal Disease Network (U54NS065768) is a part of the Rare Diseases Clinical Research Network, an initiative of the Office of Rare Diseases Research, and National Center for Advancing Translational Sciences. This consortium is funded through a collaboration between National Center for Advancing Translational Sciences, National Institute of Neurological Disorders and Stroke, and National Institute of Diabetes and Digestive and Kidney Diseases. The content is solely the responsibility of the authors and does not necessarily represent the official views of the NIH. The funders had no role in study design, data collection and analysis, decision to publish, or preparation of the manuscript. The authors thank the patients who participated in this study and their families. We thank Deeksha Bali, Catherine Rehder, and Jian Dai for providing the GAA variant data and CRIM status analysis on these patients. We also thank the local clinical care teams, including M. Valerie Marrero-Stein, Matthew Stein, Kathleen Crosby, Jennifer Q. Cook, Lorien King, Michelle Mecija, Holly Zimmerman, Lisa Read, Ashley Boerst, Geetha Puthenveetil, and David Buchbinder for their contributions toward patient care, facilitating the consent process, and assistance with data gathering.

Address correspondence to: Priya S. Kishnani, Duke University Medical Center, 905 S. LaSalle Street, GSRB1 Box 103856, 4th Floor, Durham, North Carolina 27710 USA. Phone: 919.684.2036; Email: priya.kishnani@duke.edu.

1. Porter S. Human immune response to recombinant human proteins. J Pharm Sci. 2001;90(1):1-11.

2. Banugaria SG, et al. The impact of antibodies on clinical outcomes in diseases treated with therapeutic protein: lessons learned from infantile Pompe disease. Genet Med. 2011;13(8):729-736.

3. Baert F, et al. Influence of immunogenicity on the long-term efficacy of infliximab in Crohn's disease. N Engl J Med. 2003;348(7):601-608.

4. Wadhwa M, et al. Immunogenicity of granulocyte-macrophage colony-stimulating factor (GM-CSF) products in patients undergoing combination therapy with GM-CSF. Clin Cancer Res. 1999;5(6):1353-1361.

5. Bertolotto A. Neutralizing antibodies to interferon beta: implications for the management of multiple sclerosis. Curr Opin Neurol. 2004;17(3):241-246.

6. Hjelm Skog AL, et al. Alteration of interleukin 2 (IL-2) pharmacokinetics and function by IL-2 antibodies induced after treatment of colorectal carcinoma patients with a combination of monoclonal antibody 17-1A, granulocyte macrophage colony-stimulating factor, and IL-2. Clin Cancer Res. 2001;7(5):1163-1170.

7. van Gelder CM, Hoogeveen-Westerveld M, Kroos MA, Plug I, van der Ploeg AT, Reuser AJ. Enzyme therapy and immune response in relation to CRIM status: the Dutch experience in classic infantile Pompe disease. J Inherit Metab Dis. 2015;38(2):305-314.

8. Kishnani PS, et al. A retrospective, multinational, multicenter study on the natural history of infantile-onset Pompe disease. J Pediatr. 2006;148(5):671-676.

9. Nicolino M, et al. Clinical outcomes after long-term treatment with alglucosidase alfa in infants and children with advanced Pompe disease. Genet Med. 2009;11(3):210-219.

10. Kishnani PS, et al. Chinese hamster ovary cell-derived recombinant human acid alpha-glucosidase in infantile-onset Pompe disease. J Pediatr. 2006;149(1):89-97.

11. Kishnani PS, et al. Recombinant human acid [alpha]-glucosidase: major clinical benefits in infantile-onset Pompe disease. Neurology. 2007;68(2):99-109.

12. Kishnani PS, et al. Cross-reactive immunologic material status affects treatment outcomes in Pompe disease infants. Mol Genet Metab. 2010;99(1):26-33.

13. Berrier KL, et al. CRIM-negative infantile Pompe disease: characterization of immune responses in patients treated with ERT monotherapy. Genet Med. 2015;17(11):912-918.

14. Lumizyme R [Package Insert]. Cambridge, MA: Genzyme Corporation, 2016

15. Doerfler PA, Nayak S, Herzog RW, Morel L, Byrne BJ. BAFF blockade prevents anti-drug antibody formation in a mouse model of Pompe disease. Clin Immunol. 2015;158(2):140-147.

16. Ohashi T, et al. Administration of anti-CD3 antibodies modulates the immune response to an infusion of $\alpha$-glucosidase in mice. 
Mol Ther. 2012;20(10):1924-1931.

17. Sun B, et al. Non-depleting anti-CD4 monoclonal antibody induces immune tolerance to ERT in a murine model of Pompe disease. Mol Genet Metab Rep. 2014;1:446-450.

18. Joly MS, et al. Transient low-dose methotrexate generates B regulatory cells that mediate antigen-specific tolerance to alglucosidase alfa. J Immunol. 2014;193(8):3947-3958.

19. Elder ME, et al. B-Cell depletion and immunomodulation before initiation of enzyme replacement therapy blocks the immune response to acid alpha-glucosidase in infantile-onset Pompe disease. J Pediatr. 2013;163(3):847-54.e1.

20. Lim HH, et al. Immunomodulation to enzyme replacement therapy with tolerogenic nanoparticles containing rapamycin in a murine model of Pompe disease. Mol Genet Metab. 2017;120(1):S83-S84.

21. Mendelsohn NJ, Messinger YH, Rosenberg AS, Kishnani PS. Elimination of antibodies to recombinant enzyme in Pompe's disease. NEngl J Med. 2009;360(2):194-195.

22. Messinger YH, et al. Successful immune tolerance induction to enzyme replacement therapy in CRIM-negative infantile Pompe disease. Genet Med. 2012;14(1):135-142.

23. Banugaria SG, et al. Algorithm for the early diagnosis and treatment of patients with cross reactive immunologic material-negative classic infantile Pompe disease: a step towards improving the efficacy of ERT. PLoS One. 2013;8(6):e67052.

24. Banugaria SG, et al. Bortezomib in the rapid reduction of high sustained antibody titers in disorders treated with therapeutic protein: lessons learned from Pompe disease. Genet Med. 2013;15(2):123-131.

25. Kazi ZB, et al. Durable and sustained immune tolerance to ERT in Pompe disease with entrenched immune responses. JCI Insight. 2016;1(11):e86821.

26. Stenger EO, Kazi Z, Lisi E, Gambello MJ, Kishnani P. Immune tolerance strategies in siblings with infantile Pompe disease-advantages for a preemptive approach to high-sustained antibody titers. Mol Genet Metab Rep. 2015;4:30-34.

27. Kishnani PS, et al. Immune response to enzyme replacement therapies in lysosomal storage diseases and the role of immune tolerance induction. Mol Genet Metab. 2016;117(2):66-83.

28. Bali DS, et al. Predicting cross-reactive immunological material (CRIM) status in Pompe disease using GAA mutations: lessons learned from 10 years of clinical laboratory testing experience. Am J Med Genet C Semin Med Genet. 2012;160C(1):40-49.

29. Mutation in Human Acid Alpha-Glucosidase. The Erasmus University Medical Center. http://cluster15.erasmusmc.nl/klgn/ pompe/mutations.html?lang=en. Updated May 2016. Accessed June 12, 2017.

30. GAA Mutation Database. Duke University. https://pediatrics.duke.edu/sites/pediatrics.duke.edu/files/field/attachments/ GAA_mutation_database.pdf. Accessed June 12, 2017.

31. Vogel M, Staller W, Bühlmeyer K. Left ventricular myocardial mass determined by cross-sectional echocardiography in normal newborns, infants, and children. Pediatr Cardiol. 1991;12(3):143-149.

32. Prater SN, Banugaria SG, Morgan C, Sung CC, Rosenberg AS, Kishnani PS. Letter to the Editors: Concerning "CRIM-negative Pompe disease patients with satisfactory clinical outcomes on enzyme replacement therapy" by Al Khallaf et al. J Inherit Metab Dis. 2014;37(1):141-143.

33. Broomfield A, et al. Response of 33 UK patients with infantile-onset Pompe disease to enzyme replacement therapy. $J$ Inherit Metab Dis. 2016;39(2):261-271.

34. França MM, Arnhold IJ. Clarification of intellectual abilities in patients with GLI2 mutations cited by Kevelam et al., 2012 Am J Med Genet Part A. Am J Med Genet A. 2012;158A(6):1519.

35. Khallaf HHA, Propst J, Geffrard S, Botha E, Pervaiz MA. CRIM-negative Pompe disease patients with satisfactory clinical outcomes on enzyme replacement therapy. JIMD Rep. 2013;9:133-137.

36. Rohrbach M, et al. CRIM-negative infantile Pompe disease: 42-month treatment outcome. J Inherit Metab Dis. 2010;33(6):751-757.

37. Rezvani AR, Maloney DG. Rituximab resistance. Best Pract Res Clin Haematol. 2011;24(2):203-216.

38. Schwab I, Nimmerjahn F. Intravenous immunoglobulin therapy: how does IgG modulate the immune system? Nat Rev Immunol. 2013;13(3):176-189.

39. Determination of cross-reactive immunological material (CRIM) status and longitudinal follow-up of individuals with Pompe disease; NCT01665326. ClinicalTrials.gov. https://clinicaltrials.gov/ct2/show/NCT01665326. Accessed June 22, 2017.

40. Kishnani PS, et al. Early treatment with alglucosidase alpha prolongs long-term survival of infants with Pompe disease. Pediatr Res. 2009;66(3):329-335.

41. Amalfitano A, et al. Recombinant human acid alpha-glucosidase enzyme therapy for infantile glycogen storage disease type II: results of a phase I/II clinical trial. Genet Med. 2001;3(2):132-138.

42. Young SP, et al. Long-term monitoring of patients with infantile-onset Pompe disease on enzyme replacement therapy using a urinary glucose tetrasaccharide biomarker. Genet Med. 2009;11(7):536-541.

43. Kaplan EL, Meier P. Nonparametric Estimation from Incomplete Observations. J Am Stat Assoc. 1958;53(282):457-481. 